\title{
Leptomeningeal Enhancement and Cortical Hypodensity in a Patient with Metastatic Endometrial Cancer: Metastasis or Stroke?
}

\section{Rachumalla PR ${ }^{1}$, Faour $A^{2}$, Harrison $M^{1}$ and Moylan E ${ }^{1 *}$ \\ ${ }^{1}$ Department of Medical Oncology, Liverpool Hospital, Australia \\ ${ }^{2}$ Department of Cardiology, Liverpool Hospital, Australia *Corresponding author: Moylan E, Department of Medical Oncology, Liverpool Hospital, Elizabeth St, Liverpool, Sydney, NSW 2170, Australia}

Received: September 04, 2018; Accepted: September 25, 2018; Published: October 02, 2018

\begin{abstract}
We report a 46-year-old woman with advanced endometrial cancer who presented to the emergency department with confusion, worsening ascites and symptoms of a lower respiratory tract infection. She was thought to have brain metastases based on a CT brain but was later identified to have multiple cerebral infarcts on an MRI of the brain secondary to Non-Bacterial Thrombotic Endocarditis (NBTE). Her anticoagulation was optimized to Enoxaparin 1mg/ $\mathrm{kg}$ twice a day. She passed away within 3 months with a recurrent ischemic stroke despite optimal anticoagulation. This case highlights the importance of accurate diagnosis of brain lesions in cancer patients and the need for proactive investigations to look for source of arterial emboli in patients with advanced malignancy. The case also demonstrates high risk of recurrent embolization and poor overall survival associated with NBTE.
\end{abstract}

Keywords: Non-Bacterial Thrombotic Endocarditis (NBTE); Marantic Endocarditis; Cancer; Metastases; Hypercoagulability

\section{Abbreviations}

CAP: Chest Abdomen and Pelvis; CT: Computed Tomography; CVA: Cerebral Vascular Accident; DVT: Deep Vein Thrombus; ECHO: Echocardiogram; MRI: Magnetic Resonance Imaging; NBTE: Nonbacterial Thrombotic Endocarditis; PE: Pulmonary Embolism; USA: United States of America; USS: Ultrasound Scans

\section{Case Presentation}

A 46-year-old woman was diagnosed with an advanced endometrial cancer and bilateral pulmonary emboli 5 months prior to this presentation. Histopathology on a pipelle biopsy of the endometrium was reported to show a grade 1 , ER $>60 \%$ positive, $\mathrm{PR}>60 \%$ positive endometriosis adenocarcinoma. The tumour was reported to demonstrate compact glands showing focal squamous differentiation and extensive mucinous differentiation with copious extracellular mucin. The tumour stroma contained abundant foamy macrophages. The patient received 3 cycles of Carboplatin and Paclitaxel from April to June 2017 with a significant clinical response, but she had discontinued treatment prematurely following initial improvement. She presented to the emergency department in September 2017 with recent-onset confusion, worsening ascites and lower respiratory tract symptoms. She had no significant comorbidities. She was on enoxaparin $1.5 \mathrm{mg} / \mathrm{kg}$ per day for pulmonary emboli although adherence to prescribed therapy was unable to be confirmed. She was a non-smoker and had no significant medical family history. She was commenced on empirical antibiotic therapy for presumed respiratory tract infection with little improvement in her symptoms. Her CA 125 was increasing compared to previous readings. The CA125 level at the end of chemotherapy was 780 $\mathrm{kU} / \mathrm{L}$ (normal range: $0-35 \mathrm{kU} / \mathrm{L}$ ) and was $2693 \mathrm{kU} / \mathrm{L}$ at the time of presentation to emergency department. She had a re-staging CT

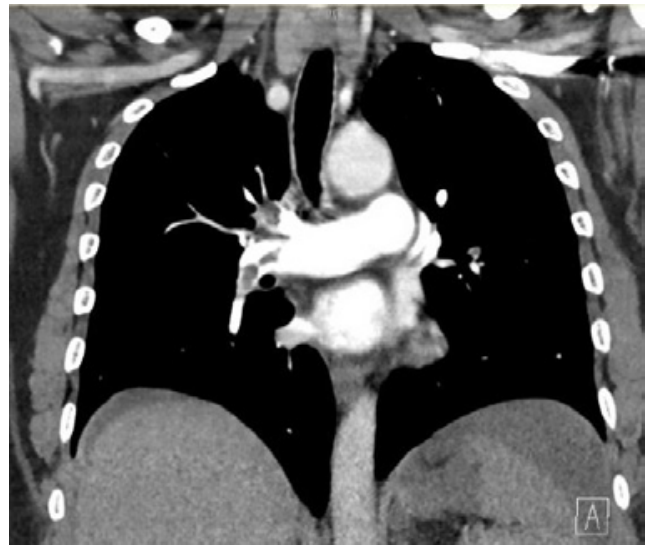

Figure 1A: Right pulmonary embolism.

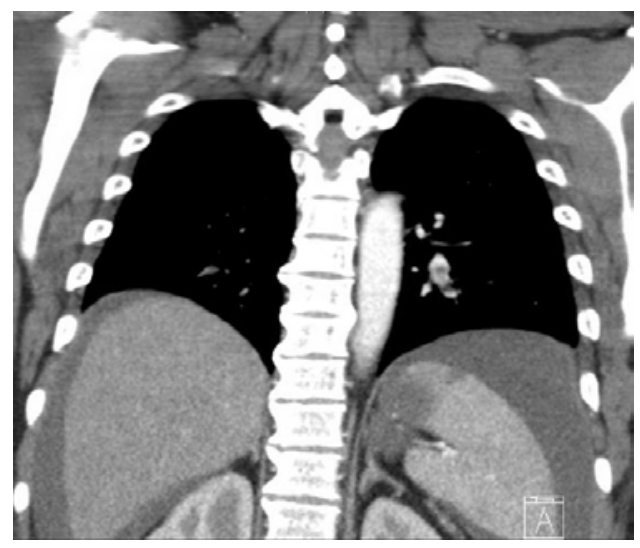

Figure 1B: Left pulmonary embolism.
Ann Hematol Oncol - Volume 5 Issue 6 - 2018

ISSN : 2375-7965 | www.austinpublishing group.com

Moylan et al. (C) All rights are reserved
Citation: Rachumalla PR, Faour A, Harrison M and Moylan E. Leptomeningeal Enhancement and Cortical Hypodensity in a Patient with Metastatic Endometrial Cancer: Metastasis or Stroke?. Ann Hematol Oncol. 2018; 5(6): 1215. 


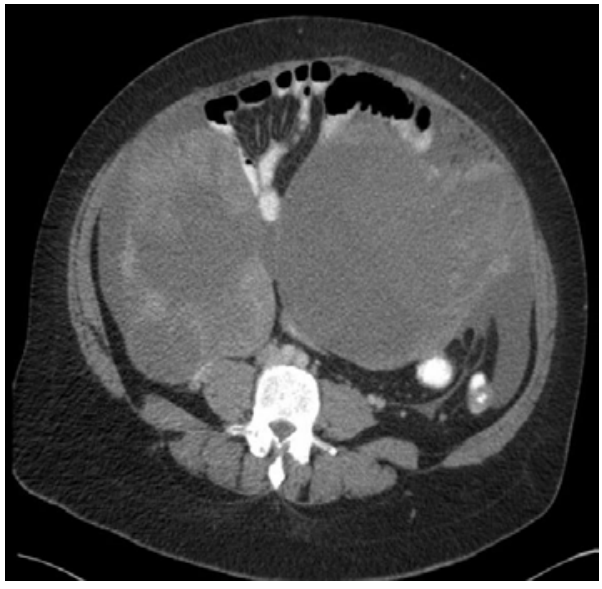

Figure 2: Abdominal solid/cystic mass.

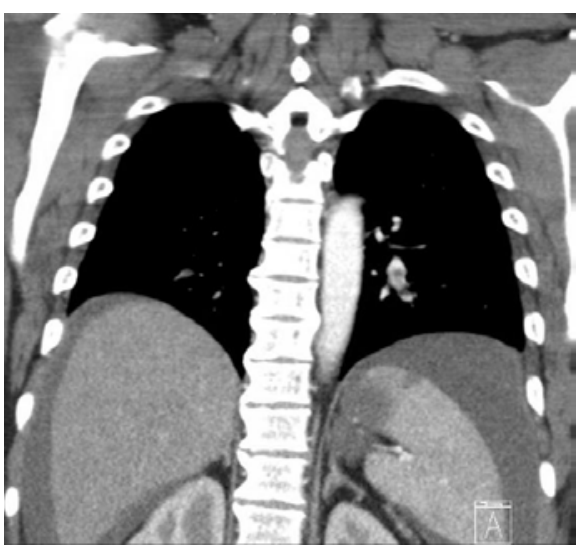

Figure 3: Splenic infarct.

CAP and cerebral CT. CT CAP showed recurrent pulmonary emboli (Figures 1A \& 1B) progression of cancer (Figure 2) with increase in the size of pelvic mass, omental caking, large volume ascites, extensive abdominal lymphadenopathy and new lung base/diaphragmatic pleural metastases. There was also a wedge shaped splenic infarct (Figure 3). A contrast-enhanced CT brain was reported to show leptomeningeal enhancement (Figure 4) associated with a wedge shaped area of hypodensity involving the left parietal cortex and subcortical white matter. This raised the possibility of multiple brain metastases. Further evaluation with MRI of the brain (Figures 5 \& 6) showed multifocal acute and subacute infarcts in both cerebrum and cerebellum. These findings led to further investigations in order to identify the source of emboli, suspecting either the presence of a patent foramen ovale with paradoxical systemic emboli or marantic endocarditis. A transoesophageal echocardiogram was reported to show a mobile right ventricular density (Figure 7) and a mobile anterior mitral leaflet density (Figure 8). Three peripheral sets of blood cultures were negative for organisms and therefore, a diagnosis of marantic endocarditis was made. The anticoagulation was changed from Enoxaparin $1.5 \mathrm{mg} / \mathrm{kg}$ daily to $1 \mathrm{mg} / \mathrm{kg}$ twice a day. The patient was also recommenced on Carboplatin/Paclitaxel chemotherapy for her underlying malignancy. Her symptoms slightly improved but she passed away 10 weeks later following another ischemic stroke.

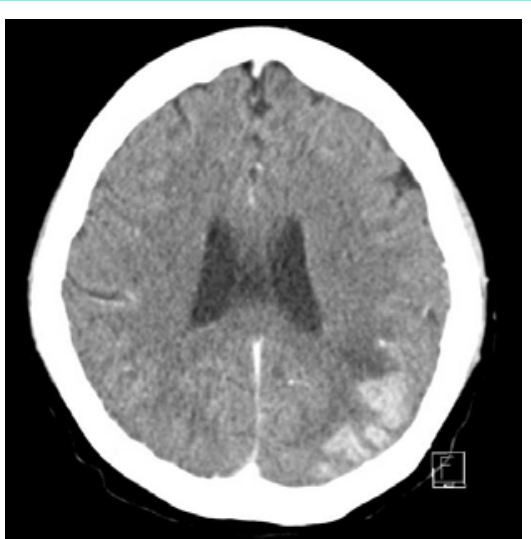

Figure 4: Leptomeningeal enhancement on cerebral CT.

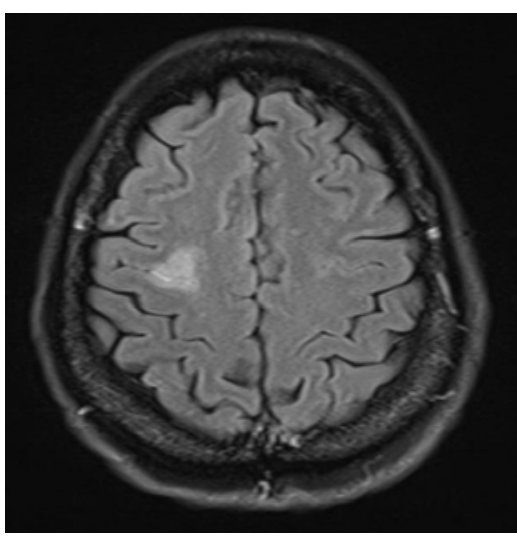

Figure 5: Right frontal infarct on MRI of Brain.

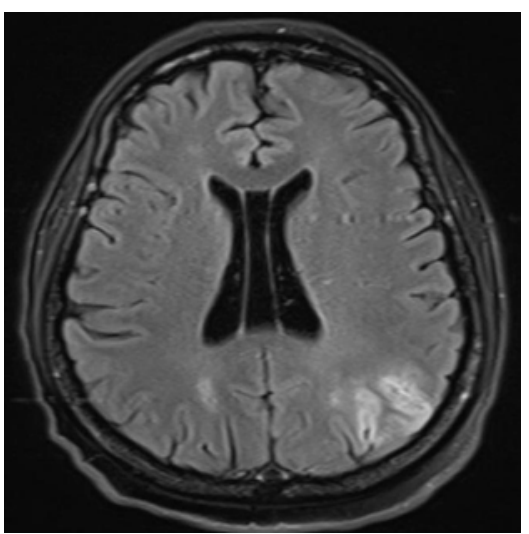

Figure 6: Parietal infarcts on MRI of Brain.

\section{Discussion}

Sterile thrombi deposits on the heart valves characterize nonbacterial thrombotic endocarditis (also called marantic endocarditis, Libman-Sacks endocarditis or verrucous endocarditis). The pathogenesis of NBTE is unclear but circulating cytokines and tumour necrosis factor-related endothelial injury is believed to predispose to thrombi formation [1]. The valvular vegetations have been reported to be mostly left sided in a large case series of 171 autopsies [2,3]. However, the presence of right-sided valvular 


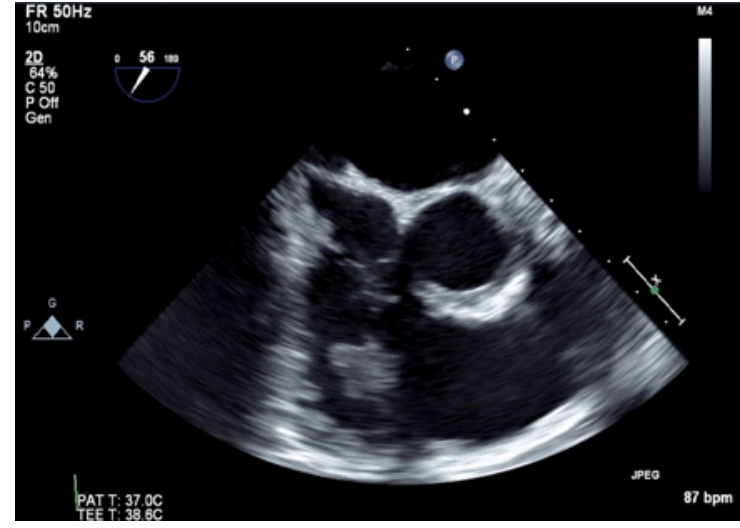

Figure 7: Right ventricular thrombus in transit (4 cm in size).

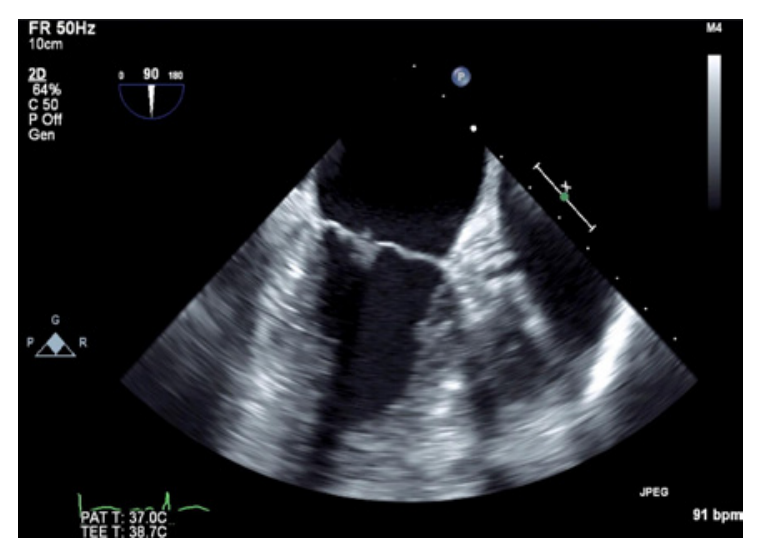

Figure 8: Mitral valve mobile density (2 $\mathrm{mm}$ in size).

vegetations in a patient with no significant risk factors, such as intravenous drug use, should raise the suspicion of NBTE.

Non-bacterial thrombotic endocarditis is commonly a postmortem diagnosis with low incidence of less than $2 \%$ of autopsies [1-6]. However, the high degree of clinical suspicion in our patient has helped to make an early diagnosis. Cancer accounts for 18 $80 \%$ cases of NBTE $[2,5,7,8]$. Cancer is a hypercoagulable state and almost $15 \%$ of patients suffer a thromboembolic event during their lifetime $[1,9,10]$. This patient also had bilateral PE at the time of cancer diagnosis which could have originated either from a DVT (USS of legs was not performed) or a right ventricular thrombus that was later seen at the time of diagnosis of NBTE. In a large series of pancreatic cancers, mucinogenic tumors had higher association with Trousseau's syndrome [11]. A retrospective study at Memorial Sloan-Kettering, USA has also showed increased association of NBTE with adenocarcinomas [12]. Our patient's omental and endometrial biopsies showed adenocarcinoma consistent with the above reports. However, NBTE and strokes are infrequent in gynecological cancer [13].

NBTE vegetations tend to embolise more frequently compared to infective vegetations, possibly due to less inflammation and weak attachment to heart valves [14]. NBTE is associated with higher incidence of cerebrovascular and fatal embolic coronary events in contrast to culture positive endocarditis, which is associated with higher incidence of cutaneous and septic pulmonary emboli [15]. Systemic embolism occurred in $41 \%$ of patients in case series of 171 cases of NBTE [2]. In the same series pulmonary embolism was noted in $43 \%$ of NBTE patients. The survival is lower in patients with cancer associated NBTE $[8,12,16]$. The rate of recurrent stroke is also higher in NBTE $[8,16]$. All these features of NBTE are well demonstrated in our patient's case. She passed away after a recurrent ischaemic stroke in 10 weeks after the diagnosis of NBTE.

\section{Conclusion}

This case highlights the importance of accurate diagnosis of brain lesions and proactive investigations to look for source of emboli in patients with advanced malignancy. The case also demonstrates high risk of recurrent embolization and poor overall survival of patients with NBTE in the setting of advanced malignancy.

\section{References}

1. El-Shami K, Griffiths E, Streiff M. Nonbacterial thrombotic endocarditis in cancer patients: Pathogenesis, diagnosis, and treatment. Oncologist. 2007; 12: 518-523.

2. Steiner I. [Nonbacterial thrombotic endocarditis--a study of 171 case reports]. Ceskoslovenska patologie. 1993; 29: 58-60.

3. Ojeda V, Frost F, Mastaglia F. Non-bacterial thrombotic endocarditis associated with malignant disease: a clinicopathological study of 16 cases. The Medical journal of Australia. 1985; 142: 629-631.

4. Llenas-Garcia J, Guerra-Vales JM, Montes-Moreno S, Lopez-Rios F, Castelbon-Fernandez FJ, Chimeno-Garcia J. [Nonbacterial thrombotic endocarditis: clinicopathologic study of a necropsy series]. Revista espanola de cardiologia. 2007; 60: 493-500.

5. Gonzalez Quintela A, Candela MJ, Vidal C, Roman J, Aramburo P. Nonbacterial thrombotic endocarditis in cancer patients. Acta cardiologica. 1991; 46: 1-9.

6. Deppisch LM, Fayemi AO. Non-bacterial thrombotic endocarditis: clinicopathologic correlations. American heart journal. 1976; 92: 723-729.

7. Hwang JW, Park SJ, Park SW, Chang SA, Lee SC, Lee SY. Clinical characteristics and diagnosis of non-bacterial thrombotic endocarditis. European Heart Journal Cardiovascular Imaging. 2017; 18 (Supplement 3):iii53.

8. Topcuoglu M, Kursun O, Karatas H, Buonanno F, Singhal A. Stroke in non-bacterial thrombotic endocarditis: 31-year experience. Neurology Conference: $65^{\text {th }}$ American Academy of Neurology Annual Meeting San Diego, CA United States Conference Publication. 2013; 80 (1 Meeting Abstracts).

9. Lobo Ferreira T, Alves R, Judas T, Delerue MF. Marantic endocarditis and paraneoplastic pulmonary embolism. BMJ case reports. 2017; 2017.

10. Devulapalli S, Pinto N, Gandothra C, Jayam-Trouth A, Kurukumbi M. A rare case of occipital stroke as a consequence of nonbacterial thrombotic endocarditis in ovarian clear cell carcinoma: A case report. Case Reports in Neurology. 2012; 4: 84-91.

11. Pinzon R, Drewinko B, Trujillo JM, Guinee V, Giacco G. Pancreatic carcinoma and Trousseau's syndrome: experience at a large cancer center. Journal of clinical oncology: official journal of the American Society of Clinical Oncology. 1986; 4: 509-514.

12. Singer S, Stone J, Merkler A, Cheng N, Kamel H, DeAngelis L, et al. Marantic Endocarditis (ME) markedly reduces survival in cancer patients with ischemic stroke. Neurology Conference: $65^{\text {th }}$ American Academy of Neurology Annual Meeting San Diego, CA United States Conference 
Publication. 2013; 80 (1 Meeting Abstracts).

13. Schabas A, Teal P, Yip S, Mann S. A case series of non-bacterial thrombotic endocarditis associated with gynecological malignancies. Neurology Conference: $66^{\text {th }}$ American Academy of Neurology Annual Meeting, AAN. 2014; 82 (10 SUPPL. 1).

14. Roldan CA, Sibbitt WL, Qualls CR, Jung RE, Greene ER, Gasparovic CM, et al. Libman-Sacks endocarditis and embolic cerebrovascular disease. JACC Cardiovascular imaging. 2013; 6: 973-983.
15. Yusuf SW, Ali SS, Swafford J, Durand JB, Bodey GP, Chemaly RF, et al. Culture-positive and culture-negative endocarditis in patients with cancer: A retrospective observational study, 1994-2004. Medicine. 2006; 85: 86-94.

16. Yang JC, Chuy KL, Yu A, Mantha S, Steingart R, Gupta D. Epidemiology and outcomes of transesophageal echocardiogram confirmed nonbacterial thrombotic endocarditis in patients with malignancy with embolic cerebrovascular accidents. Journal of the American Society of Echocardiography. 2018; 31: B19.
Ann Hematol Oncol - Volume 5 Issue 6 - 2018

ISSN : 2375-7965 | www.austinpublishing group.com

Moylan et al. (C) All rights are reserved
Citation: Rachumalla PR, Faour A, Harrison M and Moylan E. Leptomeningeal Enhancement and Cortical Hypodensity in a Patient with Metastatic Endometrial Cancer: Metastasis or Stroke?. Ann Hematol Oncol. 2018; 5(6): 1215. 